\title{
Management of concurrent severe preeclampsia and thrombotic thrombocytopenic purpura (TTP)
}

\begin{abstract}
Thrombotic thrombocytopenic purpura (TTP) is a devastating disease involving the micro circulating (capillary wall damage) of multiple organs and could be seriously fatal if not diagnosed and treated promptly. 1 TTP is a rare complication of pregnancy with a poor prognosis and high fetal mortality, especially when it occurs during the first trimester.2 But most of TTP cases occur in the late trimester of pregnancy. Untreated TTP is associated with more than $90 \%$ mortality rate.3 Plasma replacement, even in pregnancy, remains as the corner stone of prevention and treatment of TTP, especially those with autoantibody- driven ADAMTS-13-deficient TTP.4
\end{abstract}

Keywords: complication, treatment, proteinuria, hypermagnesaemia, epigastric
Volume 6 Issue I - 2018

\author{
Leila Kheirkhah,' Sadegh asoubar, ${ }^{2}$ Amir \\ Hossein $\mathrm{Abdi}^{3}$ \\ 'Department of Anesthesiology and Intensive Care,Alborz \\ hospital, Karaj, Iran \\ 2Department of Internal Medicine, Alborz hospital, Karaj, Iran \\ ${ }^{3} \mathrm{MSN}$ of Nursing, Alborz hospital, Karaj, Iran
}

\section{Correspondence: Leila kheirkhah, Department of Anesthesiology and Intensive Care,Alborz hospital, Karaj, Iran, Tel 00989126649II2, Email drleilakheirkhah@gmail.com}

Received: December 15, 2017| Published: February 14, 2018
Abbreviations: SLE, systemic lupus erythematous; APS, anti phospholipid syndrome; AFLP, cute fatty liver during pregnancy; TMA, thrombotic microangio pathies; TTP, thrombotic thrombocytopenic purpura; HUS, hemolytic uremic syndrome

\section{Case report}

A 24- year- old parturient G2 P1 A0 at the gestational age of 38 weeks, presented at emergency department. She had a history of proteinuria (3+) eight months prior to admission in our hospital. Because of severe high blood pressure $220 / 160 \mathrm{mmHg}$, proteinuria $(3+)$, high serum creatinine (cr.=1.7), mild loss of consciousness and she was admitted in intensive care unit. After some medical treatment (magnesium sulfate and hydralazine) and partially stabilization of her blood pressure, urgent pregnancy termination via lower segment caesarean section (LSCS) was performed. On admission in ICU (the first day) she had no headache and blurred vision, but complained of epigastric pain and nausea. Blood sugar was normal vital signs: BP:220/160mmHg, PR:90/min, RR:18/min, T:37.8 C., spo2>92\% (by room air).

Coagulation tests (PT, PTT and INR) were all within normal limits on the admission time and all over the course of hospitalization. Urine analysis showed $\mathrm{RBC}=3-4$ and proteinuria $(3+)$. The cell blood count showed result: WBC:13000, Hb:11.5, Hct:35, MCV:89.75, PLT:181000, RDW:13.2.

Blood pressure was controlled by hydralazine $5 \mathrm{mg}$, IV/repeated doses and methyldopa $250 \mathrm{mg} / \mathrm{q} 4 \mathrm{~h}$ PO. Labetalol was not with reach at that time. Mgso4 was given promptly as a 4 gr loading dose intra venous, followed by a maintenance intravascular infusion of 1 to $2 \mathrm{gr} / \mathrm{h}$ by controlled infusion pump. It lasted just for 24 hours and at the rest of the course of treatment we had to discontinue Mgso4 infusion because of severe hypermagnesaemia. On the day two of ICU admission (one day after caesarean section) the patient had hemoptysis, thrombocytopenia (PLT:56000) hyperkalemia (k:6), high lactate dehydrogenase (LDH:3125), Hyperbilirubinemia (Bill Total:3.3;Bill Direct:1.3), peripheral blood smear showed anisocytosis, Burr cell, Roulex formation mild increase of liver function test
(AST $=47, \mathrm{ALT}=80$ ), negative blood cultures after 48 hours, decrease of $\mathrm{Hb}$ up to $8.5 \mathrm{mg} \%$, rise of creatinine up to 3 and more, mild loss of consciousness; were all in favor of HELLP syndrome or TTP concomitant with severe preeclampsia. According to hematologic consultation, our patient was supposed to undergo plasma exchange after verifying the existence of schistocytes in peripheral blood smear. Hence, plasma exchange was requested for the patient straight after PBS examination. D-Dimer $>10000$, FDP $>20$, Serum Fibrinogen: 165 .

Unfortunately 5 units of platelets and 10 units of cryoprecipitate were transfused to the patient soon after $\mathrm{C} / \mathrm{S}$ in recovery room. Furthermore thrombocytopenia started to become worse. On days three and four of hospitalization we were facing additional problems, making the current condition worse, these were oliguria, profound thrombocytopenia PLT. Count:25000, Fibrinogen:270, FDP $>20$, massive bloody secretions from the drain inserted in caesarean incision, refractory metabolic acidosis $(\mathrm{PH}<7.3), \mathrm{Cr}>3$ and all findings in favor of acute kidney injury(AKI)-both in laboratory tests and ultrasonographic examination- and also gross hematuria, hyermagnesemia and severe ileus resulted in abdominal distension and respiratory distress( Ileus treatment was performed immediately). Liver function tests (ALT\&AST) were increased up to 94. In this new condition a shaldon catheter was placed in right subclavian vein and the patient underwent hemodialysis. On day three we had a report of slight amount of schistocyte and $(+1)$ on the next day, hence, plasma exchange was performed for her on a daily basis. On the other side, she was on daily hemodialysis without heparin, transfusion of whole blood, FFP, Cryoprecipitate, antihypertensive (consisting of calcium channel blockers i.e. diltiazem, $\alpha$ blockers i.e. prazocin), anticonvulsives, antibiotics including ceftriaxone and clindamycin, steroid therapy, intra vascular fluid therapy. All of these were on the basis of the progress of patient's condition. On day 6patient developed polyuria urine output: $250-300 \mathrm{cc} / \mathrm{h}$. On the Other side we had a rise in platelet count up to 80000 and never decreased from that time. Bleeding from the drain lessened obviously. On day 7patient's consciousness was complete with oriented voluntary movement of limbs. At that time we had no schistocytes on PBS, decreasing of LDH less than $800(\mathrm{LDH}<800)$ and no bleeding tendency, therefore plasma exchange was discontinued. 
On day 10, after keeping tight control on fluid and electrolyte balance during polyuric phase of AKI;patient recovered her consciousness and showed a decreased in serum creatinine level and correction of acidosis. Hence, there was no more need to hemodialysis. On day 13 the patient began to breast feed her child. Plasma exchange discontinued. On day 17 there was no need to hemodialysis and the patient was good enough to be discharged from the hospital.

\section{Additional diagnostic procedures and tests}

Since the patient was too ill to be transferred to CT-scan department all diagnostic procedures were performed portable on the bedside. We had the chance of having definite serial ultrasonographic checks of abdominal and pelvic cavities indicating that we could be sure of not having any kind of abnormal internal bleeding. This fact helped our obstetrician not to perform second revision of the site of operation. Laboratory findings including: ANA, FANA, Anti dsDNA, APA, C3, C4, CH50, HBS Ag, Anti HCV Ab, Anti HIV, lupus anticoagulant, anticardiolipin and serum albumin were not abnormal. ADAMTS 13 Ag 5.22 high ADAMTS 13 auto Ab 14.4 borderline verifying in blood sample. Complications due to invasive procedures at the time of patient's presentation to hospital, she was suffering from mild to moderate respiratory distress investigation by chest $\mathrm{x}$-ray and chest sonography indicated moderate bilateral pleural effusion. In addition after replacement of shaldon catheter on the day of admission, we had right sided hemopnuemothorax leading to insertion of chest tube, on day9, chest tube discharge was less than $100 \mathrm{cc} / 24 \mathrm{~h}$ and on day 12 we removed it. Biopsy sample taken from kidney 3months after hospitalization. The glomerulus shows segmental sclerosis with synechiae to overlying, Bowman's capsule (methenamine silver, x400) (Figure 1, Figure 2 \& Figure 3).

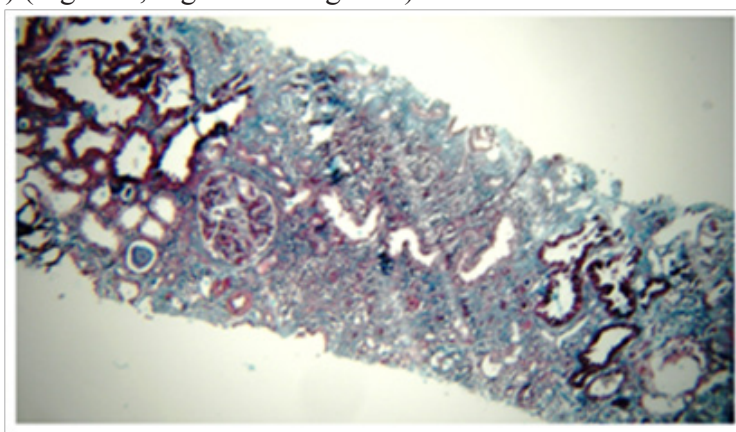

Figure I The glomerulus shows segmental sclerosis with synechiae to overlying, Bowman's capsule.

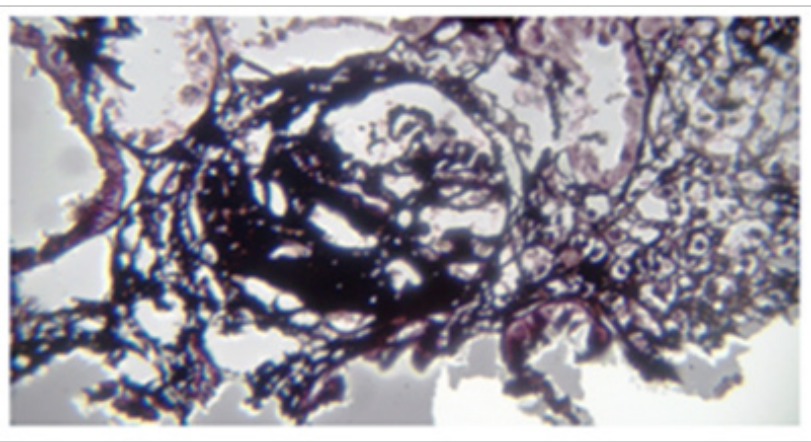

Figure 2 The glomerulus shows segmental sclerosis with synechiae to overlying, Bowman's capsule.

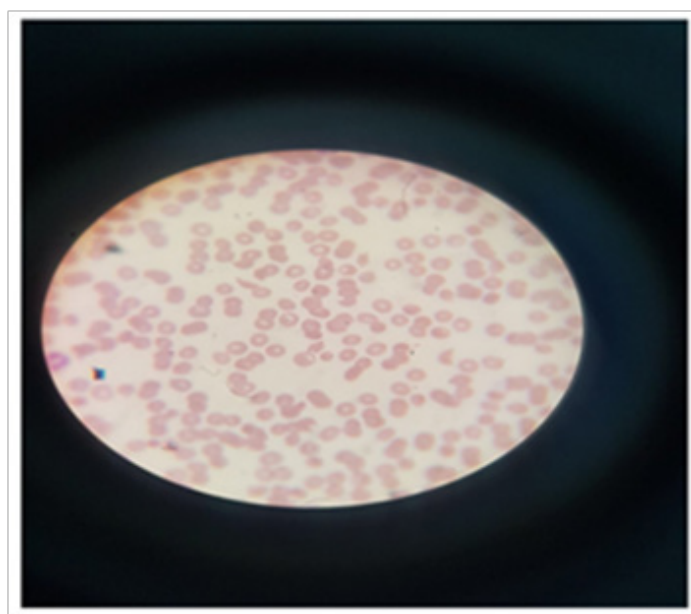

Figure 3 The glomerulus shows segmental sclerosis with synechiae to overlying, Bowman's capsule.

\section{Discussion}

There are several fatal conditions complicating preeclampsia and should be under consideration seriously, consisting of systemic lupus erythematous (SLE), anti-phospholipid syndrome (APS), acute fatty liver during pregnancy (AFLP), thrombotic microangio pathies (TMA) appearing as thrombotic thrombocytopenic purpura (TTP) or hemolytic uremic syndrome (HUS), HELLP syndrome and sever sepsis(5-7). Among these imitators of preeclampsia the differential between TMA and HELLP syndrome is really confusing and play an important role in saving the mother and her future child's lives. According to some studies the definitive marker for TTP is undetectable blood ADAMTS 13 activity..$^{5-10}$ In general terms, some complications during pregnancy including hemolysis, concurrence of arterial hypertension and proteinuria, renal failure, elevated liver enzymes, thrombocytopenia and in some cases signs of visual, neurological, pancreatic or pulmonary disturbances lead to misdiagnosis in sever preeclampsia Complicated with HELLP syndrome or with thrombotic microangiopathies (Table 1). ${ }^{11}$

Table I Clinical and Laboratory Features of TTP, HUS, and HELLP

\begin{tabular}{llll}
\hline Clinical and laboratory features & TTP & HUS & HELLP \\
\hline Neurologic sxs & +++ & $+/-$ & $+/-$ \\
Fever & ++ & $+/-$ & - \\
Hypertension & $+/-$ & $+/-$ & $+/-$ \\
Renal dysfunction & $+/-$ & +++ & $+/-$ \\
Skin lesions-purpura & + & - & - \\
Platelets & $\downarrow \downarrow \downarrow$ & $\downarrow \downarrow$ & $\downarrow$ \\
$\begin{array}{l}\text { Prothrombin time (PT)/activated partial } \\
\text { thromboplastin time (aPTT) }\end{array}$ & $\Leftrightarrow$ & $\Leftrightarrow$ & $\uparrow$ or $\Leftrightarrow$ \\
$\begin{array}{l}\text { Fibrinogen } \\
\text { BUN/creatinine }\end{array}$ & $\Leftrightarrow$ & $\Leftrightarrow$ & $\downarrow$ or $\Leftrightarrow$ \\
$\begin{array}{l}\text { Aspartate aminotransferase (AST)/alanine } \\
\text { aminotransferase (ALT) }\end{array}$ & $\Leftrightarrow$ & $\Leftrightarrow \uparrow \uparrow$ & $\uparrow$ or $\Leftrightarrow$ \\
& & & $\uparrow$ \\
Lactate dehydrogenase (LDH) & $\uparrow$ & $\uparrow \uparrow \uparrow$ & $\uparrow$ \\
\end{tabular}


In any case all appropriate treatments of preeclampsia (antihypertensive- magnesium sulfate) and other imitators of it should be commenced without delay. ${ }^{12}$ Since TTP has got a high mortality rate if not treated immediately, once the diagnosis of it is certain or even highly suspected, plasma exchange should be considered as the matter of great urgency, often in conjunction with steroid, antibiotics, when there are clues of sever sepsis. ${ }^{3,13}$ Platelet infusion should be avoided, even in cases of profound thrombocytopenia, as it could worsen the situation and lead to sever cardiovascular events in TTP. ${ }^{14}$ Many papers have dealt with this question that, whether sever HELLP syndrome particularly in the postpartum period is a form of TMA or not?. ${ }^{5,6,9,15-19}$ Having detailed knowledge of natural history of each disease process and the considerable overlap of them, which make them seem to be "disease syndrome" rather than single disease, will certainly help us to distinguish these related peculiar conditions. ${ }^{6}$ Considering to all mentioned above we should bear in mind not to misdiagnose TTP as HELLP syndrome. ${ }^{5,6,15}$ More over regarding to the risks of large volume plasma, plasma exchange should be requested just when there is highly suspected evidence of TTP/HUS. ${ }^{17}$ In addition to all previous concerns, the financial cost of plasma exchange constitutes a great part of its considerations. ${ }^{6,12}$ Among all similarities between HELLP syndrome and TMA, some are in favor of TMA and the others were indicating HELLP syndrome existence.

One helpful hint is that hemolysis is at foreground In TTP, HUS, leading to red-colored urine or/and resulting the need for red blood cells transfusion. On the other side, in HELLP syndrome, the intensity of hemolysis is far less than TMA. It is emphasized by Weinstein himself, that hemolysis was absent in about $30 \%$ of patients..$^{20}$ One another point in differentiation of these two conditions is profound thrombocytopenia in TMA, as low as $10-20 * 109 \mathrm{~L}^{-1}$, comparing to HELLP syndrome in which platelet count often remains more than $50 * 109 \mathrm{~L}^{-1}$, the lower amounts happen in about $15 \%$ of all patients. ${ }^{6}$ There are five groups of signs which are called the classic pentad of TMA, emphasizing that TTP is a mechanical hemolytic anemia. These are thrombocytopenia, microangiopathic hemolytic anemia (coombs negative) ruling out autoimmune hemolytic anemia-fever, neurologic disturbances and renal involvement which are fully presented in only a minority of patients. Concurrent existence of hemolytic anemia and thrombocytopenia is indicative of TTP. Three other less common classical manifestations are: renal failure, central nervous involvement and fever. ${ }^{3,21-23}$ We should keep in mind that in new cases of severe hemolysis associated with profound thrombocytopenia, laboratory data should be obtained to rule out TMA..$^{1,3,13,14}$ Recently one proposed laboratory finding for TTP is a lactate dehydrogenase (LDH) /aspartate aminotransferase (AST) ratio above 22.12. ${ }^{19}$ In the past pathological criteria were mandatory to define the disease, consisting of glomerular capillary endotheliosis associated with micro vascular platelet thrombi, fibrin formation in and around glomeruli, widespread arterial thrombosis, sub endothelial clear deposits in glomerular capillaries. ${ }^{1,3,18,23,25}$ Since a vast majority of patients show profound thrombocytopenia and critically ill there is a limited access to histological studies. Findings in favor of TTP are: micro vascular thrombi of Von Willebrand factor (WVF) and fibrin in kidney, skin or bone marrow, while fibrin thrombi alone indicates HELLP syndrome more than the other possibilities. Current trend is towards diagnosis of TTP by measuring a biological parameter (ADAMTS 13 activity), if less than $10 \%$ (in case of TMA highly imitating HELLP syndrome) is known as an index for TTP. ${ }^{3,13}$ The activity of this enzyme is low in HELLP syndrome too, but not to the same extent as in TTP. In some papers it is reported to be $31 \%$ (range $12-43 \%$ ) in HELLP syndrome, compared with $71 \%$ at the end of normal pregnancy and less than $10 \%$ in TTP. ${ }^{26,27}$

It is advisable to make treatment decision with the help of this index. ${ }^{28}$ Some other useful factors including severity of liver disturbances in comparison to hemolysis and dramatic rise in liver function tests are highly suggesting of HELLP syndrome more than the other diagnosis. On the other side data indicating mild liver involvement very high level of $\mathrm{LDH}$, elevated LDH/AST ratio and gross hematuria support the existence of TTP. ${ }^{19}$ Some researchers have said that in cases of ADAMTS13 activity within the range of usually seen at the end of pregnancy ${ }^{29,30}$ in other word mild decrease in ADAMTS13 activity allows the rule out TTP, and post-partum severe HELLP syndrome can be proposed. Hence this situation can be resolved without the need for plasma exchange, but we cannot ignore the possibility that plasma exchange may be of some benefit in postpartum severe HELLP syndrome after all. ${ }^{29-31}$ Yet, the question about plasma exchange utility in these situations ${ }^{5,17}$ remains unanswered. Very recently, there is report of nine cases successfully underwent plasmapheresis by Owens et al., those were defined as "post-partum thrombotic- angiopathic syndrome. ${ }^{32}$ ",

\section{ADAMTSI 3 testing}

TTP is a rare disease (5-10cases per million persons per year) characterized by the massive formation of platelet rich-thrombi in the microcirculation of multiple organs. ${ }^{33-34}$ In plasma of patients with recurrent TTP because of the deficiency of a cleaving protease ADAMTS13 as being responsible for the presence of ultra large (UL) multimers of Von Willebrand factor in endothelial cells, platelets and plasma. ${ }^{35}$ After stimulation of endothelial cells UL forms of VWF present in the endothelium but not in plasma in normal conditions, promote intravascular aggregation of platelets and the consequent micro vascular and mechanical hemolysis resulting in high fluid shear stress especially in the microcirculation. ${ }^{36} \mathrm{~A}$ kind of protease responsible for regulating the multimeric structure VWF, which was identified in 2001 by Zhang et al. ${ }^{37}$ In a retrospective cohort study of patient with TTP the VWF cleaving protease was found deficient. ${ }^{38,39}$ The new member of the ADAMTS (a disintegrin and metaloprotease with thrombospondin 1 repeats) family of metaloprotease was called ADAMTS13. ${ }^{34}$

\section{Classification of TTP}

TTP has two main forms congenital and acquired. The first one is due to mutations in the ADAMTS 13 gene; it is rare (1:1000000) autosomal recessive and most of the time manifests at birth or during childhood. ${ }^{40,41}$ The second one which is acquired is found in two types: immune-mediated forms, due to auto antibodies against ADAMTS $13^{42,43}$ and the other one is caused by massive endothelial stimulation with consequent release of UL VWF multimers in extremely large amount exceeding the system's ability to degrade them, though the levels of ADAMTS 13 level can be normal or mildly reduced. ${ }^{44}$ The most commonly conditions present in the immune-mediated forms, often associated with sever ADAMTS 13 deficiency (levels less than $10 \%$ of normal) are pregnancy, infections, autoimmune disease and the use of drugs consisting of ticlopidine and clopidegrol.

On the other side the most common conditions associated with the type of TTP presenting with normal or mildly reduced levels of ADAMTS 13 (greater than 10\%) are metastatic tumors, organ 
transplantation and the use of drugs such as cyclosporine, mitomycin and a-interferon. ${ }^{45}$ According to severe recent studies those kind of TTP associated with severe deficiency of ADAMTS 13 activity less mortality rate than those with detectable ADAMTS 13.46-49 By contrast, Coppo et al. ${ }^{51}$ reported much high mortality rate in patients with severe ADAMTS 13 deficiency in comparison to those with nonsevere deficiency. Higher mortality rate of patients with detectable ADAMTS 13 activity could be related to the lethal fault of their underlying diseases. Along with aforementioned studies, many researchers have been carried out to define. Define the strong relation between inhibitory anti ADAMTS 13 testing and mortality rate. Overall, we come to the conclusion that existence of anti ADAMTS 13 is associated with a worse prognosis. ${ }^{47-51}$ Moreover there are some reports of a positive correlation between high inhibitor titers and severity of clinical manifestations, treatment refractoriness and the rate of deaths. ${ }^{48,52,53}$

\section{Conclusion}

The presence of schistocytes (fragmented red blood cells) on the peripheral blood smear suggest red blood cell injury from damaged endothelium and is a characteristic feature of microangiopathic hemolytic anemia. These are coombs-negative intravascular hemolytic anemia including TTP and HUS. In these two situation coagulation factor assays (prothrombin or partial thromboplastin time, fibrinogen, D-dimer and soluble fibrin monomer) are not abnormal, at least not in the initial stages. Opposite to it there is often an acute-onset profound thrombocytopenia (plt. Count, $<20 * 109 / \mathrm{L}$ ), especially in the presence of renal, CNS involvements which should keep in mind the diagnosis of TTP, HUS and if confirmed immediate specific therapy (plasma exchange) must be performed. ${ }^{33,36,54}$ In this article we presented a case of acute TTP secondary to acquired auto antibodies complicated by severe preeclampsia treated by plasma replacement of $(40-50 \mathrm{cc} /$ kg plasma) were removed at each procedure and substituted with FFP, to replace ADAMTS 13 and clotting factors, concomitant with methylprednisolone and daily hemodialysis. Signs and symptoms of preeclampsia and TTP were treated by degrees to save the parturient and child lives. On admission time, things were too confusing to make a straight forward decision but as a matter of fact some clues consisting of worsening of thrombocytopenia due to platelet infusion on the day of $\mathrm{C} / \mathrm{S}$, detecting fragmented RBCs on PBS, dramatic rise of LDH but mild rise of LFT, concomitant with almost normal coagulation assays at the beginning of the disease helped the physicians to make the assumption of TTP existence more than other preeclampsia imitators. There are many data for and against the use of ADAMTS13 testing in differentiation of TTP and making treatment decision on the basis of it. It is suggested that the use of plasma exchange in postpartum severe HELLP syndrome is allowed just in cases such as: diagnosis of TTP/HUS is very likely (a sample for ADAMTS13 activity must be taken for documentation before the first plasma exchange) or if no improvement seen in HELLP syndrome in spite of special treatments i.e. LDH level and severe thrombocytopenia 2-3 days after delivery. Today, plasma exchange on the basis of replacement of ADAMTS13 is been known as an advanced treatment of TTP and some investigators believe that high dose intravenous methylprednisolone therapy along with plasma exchange has a great part in treatment (Figures 4 \& Figure 5).

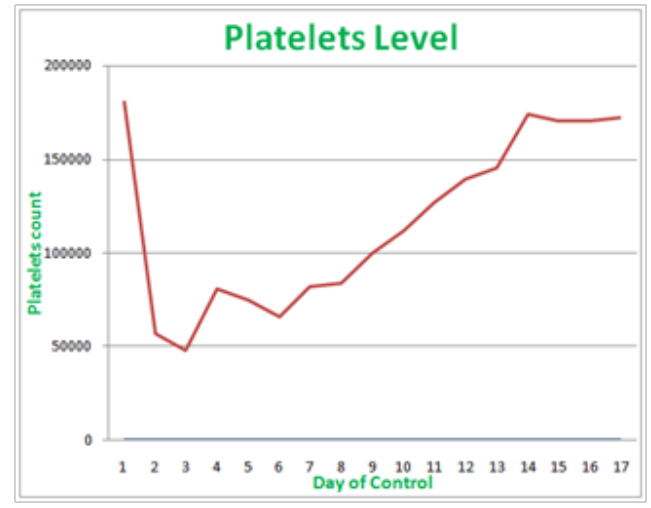

Figure 4 Platelets level.

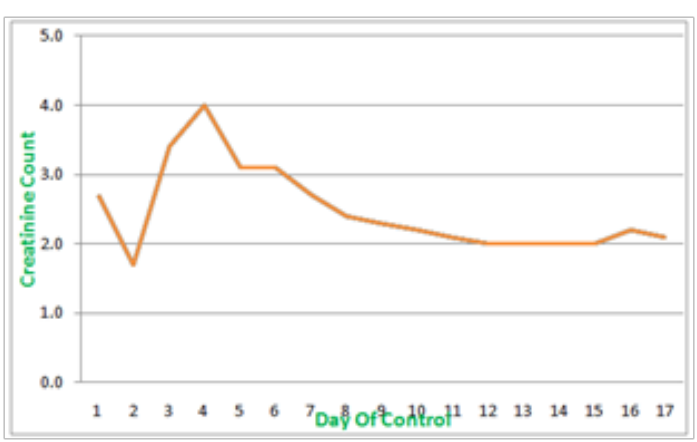

Figure 5 Creatinine level.

\section{Condensation}

In all cases of severe preeclampsia, severe HELLP syndrome and TTP, we should not hesitate for the appropriate treatment in all aspects of the disorders including preeclampsia (magnesium sulfate, antihypertensive- anticonvulsant) and the disease or in other word the micro angiopathic syndrome concomitant with it. Some findings consisting of, dramatic rise in $\mathrm{LDH}$, profound thrombocytopenia not responding to platelet infusion. Fragmented red blood cells on peripheral blood smears, ADAMTS13 assays (anti ADAMTS13 inhibitor more reliable than the other tests), mild abnormality in liver function tests, coagulation factors assays range within normal limits ( at least at the early stage of TTP); can help clinicians to distinguish TTP from HELLP syndrome. However if there is any doubt about TTP existence we should lose no time commencement of plasma exchange. Furthermore some physicians believe in adjuvant therapy by steroids or immunosuppressive agents. Still lots of encouraging studies are in process to maximize the quality of ADAMTS13 assays to define TTP.

\section{Acknowledgements}

Mojan Asadi MD, Mansoreh Rashedi MD, Sirous Talebnejad MD, Mitra Mehrazma MD, Mojgan Asgari MD, Anahid Sarkisian MD, Massoud Rahmat MD, Masoud Mohajer MD, Katayoun Ghavimi MD, Ahmad Tajmir MD, Farhad Hamzezade MD, Arsalan Fazeli MD, Sadegh Babagolzade, Azam Mahmoudi, Laila Majidi. 


\section{Conflict of interest}

The author declares no conflict of interest.

\section{References}

1. Fauci dlkaas. Harrison Principles of Internal Medicine. 19th edition. McGraw-Hill Education; 2015;2(140).

2. Mangiacapra F. Role of platelet reactivity in patients undergoing percutaneous coronary intervention. Netherlands: Technische Universitei Eindhoven; 2012

3. George JN. How I treat patients with thrombotic thrombocytopenic purpura. Blood. 2010;116(20):4060-4069.

4. Crowther MA, George JN. Thrombotic thrombocytopenic purpura: 2008 update. Cleveland Clinic journal of medicine. 2008;75(5):369.

5. Schwartz ML, Brenner WE. The obfuscation of eclampsia by thrombotic thrombocytopenic purpura. AJOG. 1978;131(1):18-24

6. Martin, Stedman CM. Imitators of preeclampsia and HELLP syndrome. Obstet Gynecol Clin North Am. 1991;18(2):181-198.

7. Sibai BM. Imitators of severe pre-eclampsia. Semin Perinatol 2009;33(3):196-205.

8. Sibai BM, Taslimi MM, El-Nazer A, et al. Maternal-perinatal outcome associated with the syndrome of hemolysis, elevated liver enzymes, and low platelets in severe preeclampsia-eclampsia. Am J Obstet Gynecol. 1986;155(3):501-509.

9. Stella CL, Dacus J, Guzman E, et al. The diagnostic dilemma of thrombotic thrombocytopenic purpura/hemolytic uremic syndrome in the obstetric triage and emergency department: lessons from 4 tertiary hospitals. Am J Obstet Gynecol. 2008;200(4):381-386.

10. Martin JN, Bailey AP, Rehberg JF, et al. Thrombotic thrombocytopenic purpura in 166 pregnancies: 1955-2006. AJOG. 2009;199(2):98-104.

11. Natalie A Bowersox. Thrombocytopenia in Pregnancy. USA: Medscape 2016

12. Pourrat O, Coudroy R, Pierre F. Differentiation between severe HELLP syndrome and thrombotic microangiopathy, thrombotic thrombocytopenic purpura and other imitators. Eur J Obstet Gynecol Reprod Biol. 2015; 189:68-72.

13. Scully M, Hunt BJ, Benjamin S, et al. Guidelines on the diagnosis and management of thrombotic thrombocytopenic purpura and other thrombotic microangiopathies. Br J Haematol. 2012;158(3):323-325.

14. Bell WR, Braine HG, Ness PM, et al. Improved survival in thrombotic thrombocytopenic purpura-hemolytic uremic syndrome: clinical experience in 108 patients. $N$ Engl J Med. 1991;325(6):398-403.

15. Knox TA, Olans LB. Liver disease in pregnancy. New England Journal of Medicine. 1996;335(8):569-576.

16. Rehberg JF, Briery CM, Hudson WT, et al. Thrombotic thrombocytopenic purpura masquerading as hemolysis, elevated liver enzymes, low platelets (HELLP) syndrome in late pregnancy. Obstet Gynecol. 2006;108(3):817820

17. Pourrat $\mathrm{O}$, Touchard $\mathrm{G}$, Robert R, et al. A kidney biopsy is clearly mandatory to confirm the indication of plasma exchanges in adult haemolytic uraemic syndrome. Ann Med Interne (Paris). 1994;145(5):369-372.

18. Fakhouri F, Vercel C, Frémeaux Bacchi V. Obstetric nephrology: AKI and thrombotic microangiopathies in pregnancy. CJASN. 2012;7(2):2100 2106.

19. Keiser SD, Boyd K, Rehberg JF, et al. A high LDH to AST ratio helps to differentiate pregnancy-associated thrombotic thrombocytopenic purpura (TTP) from HELLP syndrome. J Matern Fetal Neonatal Med. 2012;25(7):1059-1063

20. Weinstein L. Syndrome of hemolysis, elevated liver enzymes, and low platelet count: a severe consequence of hypertension in pregnancy. Am J Obstet Gynecol. 1982;142(2):159-167.

21. Samuels P, Bussel JB, Braitman LE, et al. Estimation of the risk of thrombocytopenia in the offspring of pregnant women with presumed immune thrombocytopenic purpura. N Engl J Med. 1990;323(4):229-235.

22. Amorosi EL, Ultmann JE. Thrombotic thrombocytopenic purpura: report of 16 cases and review of the literature. Medicine. 1966;45(2):139-160.

23. Rock GA, Shumak KH, Buskard NA, et al. Comparison of plasma exchange with plasma infusion in the treatment of thrombotic thrombocytopenic purpura. N Engl J Med. 1991;325(6):393-397.

24. Tsai HM. Pathophysiology of thrombotic thrombocytopenic purpura. Int J Hematol. 2010;91(1):1-19.

25. Tsai HM. Mechanisms of microvascular thrombosis in thrombotic thrombocytopenic purpura. Kidney international Supplement. 2009;75(122):S11-14.

26. Lattuada A, Rossi E, Calzarossa C, et al. Mild to moderate reduction of a von Willebrand factor cleaving protease (ADAMTS-13) in pregnant women with HELLP microangiopathic syndrome. Haematologica. 2003;88(9):1029-1034.

27. Sánchez Luceros A, Farías CE, Amaral MM, et al. Von Willebrand factorcleaving protease (ADAMTS13) activity in normal non-pregnant women, pregnant and post-delivery women. Thromb Haemost. 2004;92(6):1320 1326.

28. Pourrat O, Coudroy R, Pierre F. ADAMTS13 deficiency in severe postpartum HELLP syndrome. Br J Haematol. 2013;163(3):409-410.

29. Martin JN, Perry KG, Roberts WE, et al. Plasma exchange for preeclampsia III. Immediate peripartal utilization for selected patients with HELLP syndrome. Journal of clinical apheresis. 2004;9(3):162-165.

30. Martin JN, Files JC, Blake PG, et al. Postpartum plasma exchange for atypical preeclampsia-eclampsia as HELLP (hemolysis, elevated liver enzymes, and low platelets) syndrome. Am J Obstet Gynecol. 1995;172(4):1107-1125.

31. Martin JN, Files JC, Blake PG, et al. Plasma exchange for preeclampsia I. Postpartum use for persistently severe preeclampsia-eclampsia with HELLP syndrome. Am J Obstet Gynecol. 1990;162(1):126-137.

32. Owens MY, Martin JN, Wallace K, et al. Postpartum thrombotic microangiopathic syndrome. Transfus Apher Sci. 2013;48(1):51-57.

33. Tsai HM. Advances in the pathogenesis, diagnosis, and treatment of thrombotic thrombocytopenic purpura. $J$ Am Soc Nephrol. 2003;14(4):1072-1081.

34. Sadler JE, Moake JL, Miyata T, et al. Recent advances in thrombotic thrombocytopenic purpura. Hematology Am Soc Hematol Educ Program. 2004; 1:407-423.

35. Moake JL, Rudy CK, Troll JH, et al. Unusually large plasma factor VIII: von Willebrand factor multimers in chronic relapsing thrombotic thrombocytopenic purpura. $N$ Engl J Med. 1982;307(23):1432-1435.

36. Mannucci PM, Lavoretano S, Peyvandi F. The thrombotic microangiopathies. Blood Transfus. 2005;3:120-135.

37. Zheng X, Chung D, Takayama TK, e al. Structure of von Willebrand factorcleaving protease (ADAMTS13), a metalloprotease involved in thrombotic thrombocytopenic purpura. J Biol Chem. 2001;276(44):41059-41063. 
38. Furlan M, Robles R, Galbusera M, et al. Von Willebrand factor-cleaving protease in thrombotic thrombocytopenic purpura and the hemolyticuremic syndrome. N Engl J Med. 1998;339(22):1578-1584.

39. Tsai HM, Lian ECY. Antibodies to von Will brand factor-cleaving protease in acute thrombotic thrombocytopenic purpura. $N$ Engl $\mathrm{J} \mathrm{Med}$. 1998;339(22):1585-1594.

40. Tsai HM. Seminars in thrombosis and hemostasis. USA: Thieme Medical Publishers; 2004.

41. Levy GG, Nichols WC, Lian EC, et al. Mutations in a member of the ADAMTS gene family cause thrombotic thrombocytopenic purpura. Nature. 2001;413(6855):488-494.

42. Klaus C, Plaimauer B, Studt JD, et al. Epitope mapping of ADAMTS13 auto antibodies in acquired thrombotic thrombocytopenic purpura. Blood. 2004;103(12):4514-4519.

43. Rieger M, Mannucci PM, Hovinga JAK, et al. ADAMTS13 auto antibodies in patients with thrombotic microangiopathies and other immunomediated diseases. Blood. 2005;106(4):1262-1267.

44. Van der Plas RM, Schiphorst ME, Huizinga EG, et al. Von Will brand factor proteolysis is deficient in classic, but not in bone marrow transplantation-associated, thrombotic thrombocytopenic purpura. Blood. 2009;93(11):3798-3802.

45. Tsai HM. Current concepts in thrombotic thrombocytopenic purpura. Annu Rev Med. 2006;57:419-436.

46. Vesely SK, George JN, Lämmle B, et al. ADAMTS13 activity in thromboti thrombocytopenic purpura-hemolytic uremic syndrome: relation to presenting features and clinical outcomes in a prospective cohort of 142 patients. Blood. 2003;102(1):60-68.
47. Zheng XL, Kaufman RM, Goodnough LT, et al. Effect of plasma exchange on plasma ADAMTS13 metalloprotease activity, inhibitor level, and clinical outcome in patients with idiopathic and nonidiopathic thrombotic thrombocytopenic purpura. Blood. 2004;103(11):4043-4049.

48. Mori Y, Wada H, Gabazza EC, et al. Predicting response to plasma exchange in patients with thrombotic thrombocytopenic purpura with measurement of vWF-cleaving protease activity. Transfusion. 2002;42(5):572-580.

49. Raife T, Atkinson B, Montgomery R, et al. Severe deficiency of VWFcleaving protease (ADAMTS13) activity defines a distinct population of thrombotic microangiopathy patients. Transfusion. 2004;44(2):146-150.

50. Coppo P, Bengoufa D, Veyradier A, et al. Severe ADAMTS13 deficiency in adult idiopathic thrombotic microangiopathies defines a subset of patients characterized by various autoimmune manifestations, lower platelet count, and mild renal involvement. Medicine. 2004;83(4):233-244.

51. Böhm M, Betz C, Miesbach W, et al. The course of ADAMTS-13 activity and inhibitor titre in the treatment of thrombotic thrombocytopenic purpura with plasma exchange and vincristine. Br J Haematol. 2005;129(5):644 652 .

52. Coppo P, Wolf M, Veyradier A, et al. Prognostic value of inhibitory antiADAMTS13 antibodies in adult-acquired thrombotic thrombocytopenic purpura. British journal of haematology. 2006;132(1):66-74.

53. Tsai HM. High titers of inhibitors of von Will brand factor-cleaving metalloproteinase in a fatal case of acute thrombotic thrombocytopenic purpura. American journal of hematology. 2000;65(3):251-255.

54. Elliott MA, Nichols WL. Thrombotic thrombocytopenic purpura and hemolytic uremic syndrome (HUS). USA: Merck manual professional version; 2001. 